\title{
Unexpected features of breast cancer subtype
}

\author{
Ye-huan Liu, Ou-chen Wang*, En-dong Chen, Ye-feng Cai, Chuan-meng Pan, Fan Yang and Xiao-hua Zhang
}

\begin{abstract}
Background: Gene expression profiling of breast cancers identifies distinct molecular subtypes that affect prognosis. The aim of this study was to determine whether features of tumors especially the risks of lymph node (LN) metastases differ among molecular subtypes.

Methods: Subtypes were classified by immunohistochemical surrogates as luminal A, luminalHer2-, luminalHer2+, TNBC, and HER-2+. Data were obtained from an established, registered database of patients with invasive breast cancer treated at our hospital between July 2012 and October 2014. A total of 929 tumors were classifiable into molecular subtypes.

Results: The distribution of subtypes was luminal A (24.2\%), luminalHer2- (27.8\%), luminalHer2+ (9.1\%), TNBC $(21.3 \%)$, and HER-2+ (17.5\%). Marked differences in age, tumor size, extent of lymph node involvement, and grade were observed among subtypes. On univariate analysis, the LN positivity varied across subtypes with $33.6 \%$ in luminal A, $40.3 \%$ in luminalHer2-, $37.3 \%$ in luminalHer2+, $37.6 \%$ in TNBC, and $47.4 \%$ in HER-2+ $(p=0.201)$. There was no significant difference in LN positivity among subtypes. On multivariable analysis, grade and tumor size were independent predictors of LN positivity.

Conclusions: Predictors of LN metastases include higher grade and larger tumor size. Even though breast cancer subtype is not a statistically significant predictor of LN positivity, this information may still be useful in selecting the appropriate therapy in clinical practice.
\end{abstract}

\section{Background}

Gene expression profiling based on 496 genes has identified distinct molecular subtypes of breast cancer that differ in prognosis. Studies have demonstrated that patients with HER-2+ (human epidermal growth factor receptor-2+) and TNBC (triple negative breast cancer) tumors have poorer survival compared with patients with luminal A and luminal B (luminalHer2- and luminalHer2+) tumors and that those with luminal A tumors have a better prognosis than those with luminal $B$ tumors [1, 2]. Molecular subtype seems to be associated with the risk of local failure for patients treated with breast-conserving therapy and for those treated with mastectomy [3, 4]. However, little is known about whether the presenting characteristics especially the axillary lymph node status of breast cancer differ by molecular subtype. As we all know, nodal involvement carries important prognostic and therapeutic importance. Several predictors

\footnotetext{
* Correspondence: woc099@sina.com

Department of Oncology, The First Affiliated Hospital of Wenzhou Medical University, South of Bai-xiang Street, Ou-hai District, 325000 Wenzhou, Zhejiang, People's Republic of China
}

of lymph node metastasis have been described such as higher grade and larger tumor size $[5,6]$. However, the impact of tumor subtype on axillary status has not been well established [1,7-9].

Therefore, our purpose was to determine whether molecular subtype, as defined by estrogen receptor (ER), progesterone receptor (PR), Ki-67, and HER-2, correlates with presenting features of breast cancer, particularly to evaluate whether it is an independent predictor of axillary lymph node involvement on multivariable analysis.

\section{Methods}

This study was performed with the approval of The First Affiliated Hospital of Wenzhou Medical University Review Board. Review of a prospectively maintained database of patients evaluated and treated for breast cancer from July 2012 to October 2014 was performed. Patients between the ages of 25 and 90 years with newly diagnosed stage I-III breast cancer were identified. Of these, preoperative systemic therapy was excluded.

Patients were categorized according to tumor phenotypic subtype using the presence or absence of tumor 
makers. Specifically, they were categorized into luminal A (ER+/PR+, HER2-, Ki67 $<14 \%$ or PR $\geq 20 \%)$, luminalHer2- $(\mathrm{ER}+/ \mathrm{PR}+, \mathrm{HER} 2-, \mathrm{Ki} 67 \geq 14 \%$ or PR $<20 \%)$, luminalHer2+ (ER+ and HER-2+), TNBC (ER- and PRand HER2-), and HER-2+ (ER- and PR- and HER-2+). The criteria are recommended according to the 13th St. Gallen International Breast Cancer Conference [10, 11]. ER and PR status was determined on the basis of immunohistochemical (IHC) staining. ER/PR was conceived to be positive if the percentage of nuclear-staining cancer cells is no less than $1 \%[2,9,12]$. Tumors were considered HER-2-positive only if they were scored $3+$ by IHC or if they were HER-2-amplified (ratio $\geq 2.0$ ) on the basis of fluorescence in situ hybridization (FISH) [13, 14]. Patient and tumor characteristics evaluated included age, tumor size, grade, molecular subtype, and nodal involvement. Nodal positivity was then evaluated based on the number of tumors involving lymph nodes. A positive node was defined as a lymph node containing any cancer cells by hematoxylin and eosin stain or cytokeratin positivity via IHC. This was divided into three groups: 0 node positive, $1-3$ nodes positive, and $\geq 4$ nodes positive. Characteristics that would result in nodal positivity were then evaluated and analyzed. These included patient age, tumor size, and breast cancer subtype.
The $\chi^{2}$ test was used for binary variables and analysis of variance for continuous variables to compare the distribution of clinicopathologic characteristics among the four subtypes. All percentages and statistical tests were based on available data. Multivariate logistic regression analysis was used to determine whether subtype was independently predictive of nodal involvement after controlling for age (continuous), tumor size (continuous), and tumor grade (3 vs. 2 vs. 1). Luminal A was the reference group. Patients with missing data were excluded from multivariate analysis. All statistical tests were twosided, and a $p$ value of $<0.05$ was considered significant. All statistical analyses were performed using SAS version 9.1 (SAS Institute, Cary, NC).

\section{Results}

A total of 929 patients met the study criteria. Of these, 100 underwent breast-conserving surgery (BCS) and 829 were treated with mastectomy. The mean patient age was 52 (range, 25-90) years. Luminal A tumors were present in $24.2 \%$, luminalHer2- in $27.8 \%$, luminalHer2+ in $9.1 \%$, TNBC in $21.3 \%$, and HER-2+ in $17.5 \%$. Patient and tumor characteristics by subtype are summarized in Table 1. Among the four breast cancer subtypes, there were significant differences in the distribution of tumor

Table 1 Patient demographic and tumor data

\begin{tabular}{|c|c|c|c|c|c|c|}
\hline Feature & Luminal A & luminalHer2- & luminalHer2+ & TNBC & Her-2+ & $p$ value \\
\hline Number, n (\%) & $225(24.2)$ & $258(27.8)$ & $85(9.1)$ & $198(21.3)$ & $163(17.5)$ & \\
\hline \multicolumn{7}{|l|}{ Age at diagnosis } \\
\hline Mean \pm SD & $52.2 \pm 12.1$ & $52.3 \pm 12.5$ & $49.7 \pm 10.1$ & $52.1 \pm 11.9$ & $52.4 \pm 10.4$ & 0.433 \\
\hline \multicolumn{7}{|l|}{ Tumor size $(\mathrm{cm})$} \\
\hline Mean \pm SD & $2.0 \pm 1.1$ & $2.3 \pm 1.4$ & $2.4 \pm 1.4$ & $2.5 \pm 1.8$ & $2.5 \pm 1.5$ & 0.001 \\
\hline \multicolumn{7}{|l|}{ Size distribution } \\
\hline No. missing & 7 & 4 & 1 & 4 & 3 & \\
\hline $\mathrm{T} 1(<2 \mathrm{~cm})$ & $119(54.6)$ & $101(39.8)$ & $39(46.4)$ & $70(36.1)$ & $55(33.4)$ & 0.002 \\
\hline $\mathrm{T} 2(2 \sim 5 \mathrm{~cm})$ & $95(43.6)$ & $144(56.7)$ & $41(48.8)$ & $117(60.3)$ & $96(60.0)$ & \\
\hline $\mathrm{T} 3(>5 \mathrm{~cm})$ & $4(1.8)$ & $9(3.5)$ & $4(4.8)$ & $7(3.6)$ & $9(5.6)$ & \\
\hline \multicolumn{7}{|l|}{ Grade, $n(\%)$} \\
\hline No. missing & 19 & 25 & 6 & 15 & 11 & \\
\hline 1 & $63(30.6)$ & $28(12.0)$ & $5(6.3)$ & $9(4.9)$ & $11(7.2)$ & $<0.0001$ \\
\hline 2 & $127(61.7)$ & $166(71.2)$ & $53(67.1)$ & $80(43.7)$ & $67(44.1)$ & \\
\hline 3 & $16(7.8)$ & $39(16.7)$ & $21(26.6)$ & $94(51.4)$ & $74(48.7)$ & \\
\hline \multicolumn{7}{|l|}{ Node status } \\
\hline No. missing & 8 & 20 & 2 & 12 & 9 & \\
\hline Total positive & 73 (33.6) & $96(40.3)$ & $31(37.3)$ & 70 (37.6) & $73(47.4)$ & \\
\hline No (0) & $144(66.4)$ & $142(59.7)$ & $52(62.7)$ & $116(62.4)$ & 81 (52.6) & 0.201 \\
\hline N1 (1 3) & $48(22.1)$ & $53(22.3)$ & $15(18.1)$ & $39(21.0)$ & $39(25.3)$ & \\
\hline $\mathrm{N} 2(\geq 4)$ & 25 (11.5) & 43 (18.1) & 16 (19.3) & $31(16.7)$ & $34(22.1)$ & \\
\hline
\end{tabular}

Bold values are statistically significant $(p<0.05)$

$S D$ standard deviation 
size (all $p=0.002$ ) and grade (all $p<0.0001$ ). Luminal A tumors were smaller when compared to luminalHer2-, luminalHer2+, TNBC, and HER-2+ tumors (2.0 vs. 2.3, 2.3, 2.4, and 2.5; $p=0.001$ ). Tumors overexpressing HER-2 (luminalHer2+ and HER-2+) and TNBC subtypes were more frequently in grade 3 and T3. HER-2+ tumors were more likely to have involvement of nodes. LN metastases were detected in $343(39.1 \%)$ patients. The LN positivity rate varied across subtypes with 73 of 217 (33.6 \%) patients in luminal A, 96 of 238 (40.3\%) in luminalHer2-, 31 of 83 (37.3\%) in luminalHer2+, 70 of 186 (37.6\%) in TNBC, and 73 of 154 (47.4 \%) in HER-2+. In addition, luminal A breast cancers were more frequently nodenegative when compared to the others (66.4 vs. $59.7,62.7$, 62.4 , and $52.6 \%$, respectively) and less frequently had four or more positive nodes (11.5 vs. 18.1,19.3,16.7 and $22.1 \%$, respectively) (Fig. 1). However, on univariate analysis, these data suggest that there was no significant difference in the incidence of nodal metastases among the four breast cancer subtypes $(p=0.201)$.

On multivariate analysis, after controlling for tumor size, grade, and patient age, subtype was not a statistically significant predictor of nodal metastases $(p=0.227$ in $\geq 1$ positive $\mathrm{LN}$ and $p=0.561$ in $\geq 4$ positive $\mathrm{LN}$; Table 2). When compared to the luminal A subtype, the odds ratio for LN positivity in HER-2+ was 1.2, with $95 \%$ CI of 0.6-2.1, suggesting that HER-2+ has nodal involvement more frequently. However, none of the other subtypes was found to differ statistically significantly from the luminal A subtype in the increased risk of any nodal metastases. Furthermore, predictors of four or more positive nodes included size of the tumor of about $2 \sim 5$ and $>5 \mathrm{~cm}$ (odds ratio [OR] 2.4, 1.5-4.0, and OR $6.2,1.5-26.4)(p=0.001)$, and grade 2 or 3 tumors (OR 17.5, 2.4-130.5 and OR 22.9, 3.0-176.3) ( $p=0.015)$. Age was not associated with an increased likelihood of positive lymph nodes. Larger size and higher grade were again found to be predictive of having one or more positive nodes. In addition, when evaluating the predictors of $\geq 4$ positive nodes, tumors overexpressing HER-2 (luminalHer2+ and HER-2+) were more likely to have four or more nodes positive (OR 1.1, 0.5-2.7 and OR 1.4, 0.7-3.0) (Table 2).

\section{Discussion}

In this study, we found an unexpected result when comparing initial presenting characteristics of invasive breast cancer. On univariate analysis, factors associated with poor prognosis such as grade 3 and T3 were all far more frequent in tumors that overexpressed HER-2 and TNBC. On multivariate analysis, subtype was not a statistically significant predictor of any nodal involvement and highvolume nodal involvement (four or more positive lymph nodes). However, the HER-2+ subtype has nodal involvement more frequently when compared with the luminal A subtype.

Nodal status is an important factor associated with survival in breast cancer patients, and it is a major determinant factor in decision about therapy. Tumor size, tumor grade, tumor location, presence of lymphatic/vascular invasion, age at diagnosis, estrogen receptor status (ER), progesterone receptor status (PR), and HER-2 status have been previously published as independent variables for LN positivity [15-17]. Axillary lymph node involvement remains the most important prognostic factor in early-stage breast cancer. The observed higher frequency of node metastases in HER-2+ may account for the higher rates of local recurrence observed in HER-2-positive tumors. Nguyen et al. [3] recently studied 793 patients with invasive breast cancer of different molecular subtypes and treated with surgery and radiotherapy. The rate of local recurrence was $0.8 \%$ for the luminal A subtype, $1.5 \%$ for luminal B, $7.1 \%$ for the TNBC group, and $8.4 \%$ for the HER-2+ subtype. Even though there is no statistically significant difference among subtypes in lymph node metastases, our findings of increased nodal involvement in HER-2+ subtypes may at least partly explain the observed increase in local recurrence in the HER-2+ subtype

\section{\% Lymph Node Positivity by Subtype}

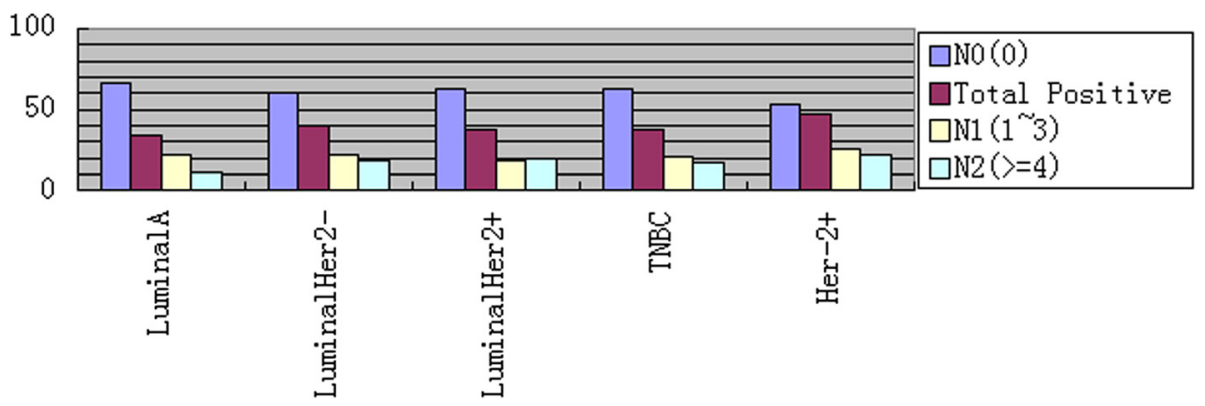

Fig. 1 Number of total positive LN by subtype ( $p=0.201)$. NO vs. N1 vs. N2. More N0 in luminal A/TNBC, more N2 in luminalHer-, luminalHer+, and Her-2+ 
Table 2 Multivariable logistic regression

\begin{tabular}{|c|c|c|c|c|}
\hline \multirow[t]{2}{*}{ Variable } & \multicolumn{2}{|c|}{$\geq 1$ Positive LN $(N=343)$} & \multicolumn{2}{|c|}{$\geq 4$ Positive LN $(N=149)$} \\
\hline & OR $(95 \% \mathrm{Cl})$ & $p$ value & OR $(95 \% \mathrm{Cl})$ & $p$ value \\
\hline Subtype ${ }^{a}$ & & 0.227 & & 0.561 \\
\hline Luminal A & 1 & & 1 & \\
\hline luminalHer2- & $0.7(0.4-1.2)$ & & $1.0(0.5-2.0)$ & \\
\hline luminalHer2+ & $0.7(0.4-1.4)$ & & $1.1(0.5-2.7)$ & \\
\hline TNBC & $0.7(0.4-1.2)$ & & $0.8(0.4-1.7)$ & \\
\hline Her-2+ & $1.2(0.6-2.1)$ & & $1.4(0.7-3.0)$ & \\
\hline Tumor size (cm) & & $<0.0001$ & & 0.001 \\
\hline$<2$ & 1 & & 1 & \\
\hline $2 \sim 5$ & $2.3(1.6-3.4)$ & & $2.4(1.5-4.0)$ & \\
\hline$>5$ & $2.7(0.7-10.2)$ & & $6.2(1.5-26.4)$ & \\
\hline Grade & & $<0.0001$ & & 0.015 \\
\hline 1 & 1 & & 1 & \\
\hline 2 & $5.6(2.8-11.2)$ & & $17.5(2.4-130.5)$ & \\
\hline 3 & $4.5(2.1-9.8)$ & & $22.9(3.0-176.3)$ & \\
\hline Age level & & 0.548 & & 0.676 \\
\hline$<50$ & 1 & & 1 & \\
\hline $50-79$ & $0.9(0.6-1.3)$ & & $1.1(0.7-1.8)$ & \\
\hline$>79$ & $2.1(0.3-13.1)$ & & $2.1(0.3-13.8)$ & \\
\hline
\end{tabular}

Bold values are statistically significant $(p<0.05)$

$L N$ lymph node, $O R$ odds ratio

aSubtypes as defined as luminal A (ER+/PR+, HER2-, Ki67 $<14 \%$ or PR $\geq 20 \%$ ), luminalHer2- (ER+/PR+, HER2-, Ki67 $\geq 14 \%$ or $\mathrm{PR}<20 \%$ ), luminalHer2+ (ER+ and HER-2+), TNBC (ER- and PR- and HER2-) and HER-2+ (ER- and PRand HER-2+)

in this study. Our data also support a previously reported study by Crabb et al. [18] that indicates that the TNBC subtype is associated with a lower incidence of axillary nodal involvement than other subtypes in four or more metastatic lymph nodes despite its poor prognosis. Meanwhile, it reported that the luminal B and HER-2+ subtypes did not predict a different risk of axillary lymph node involvement compared to the luminal A group. Our data support this finding, and we found that the HER-2+ subtype is associated with a higher likelihood of axillary metastases, despite not identifying a higher risk of having any positive nodes.

Even so, there are potential implications for the evaluation of patients for local therapy. In general, patients are fit for breast-conserving surgery with a high degree of accuracy by history, physical examination, and diagnostic mammography [19]. We found that patients overexpressing HER-2 (luminalHer2+ and HER-2+) and TNBC subtypes were more frequently in grade 3 and T3, and those overexpressing the HER-2+ subtype were more likely to have involvement of nodes, suggesting that these patients who are borderline candidates for breast conservation as a result of a large tumor size relative to the breast size would particularly likely benefit from neoadjuvant therapy that includes trastuzumab. This treatment has reported high pathologic complete response rates with the approach [20]. Discovery of nodal status before neoadjuvant therapy with axillary ultrasound and fineneedle aspiration may also be particularly profitable for the Her-2+ subtype with greater likelihood of involvement. This means most of these patients will benefit from chest wall irradiation when mastectomy is performed, and the time and cost of sentinel node biopsy could be saved even for someone who is not receiving neoadjuvant therapy.

Although the idea of using molecular subtype to select local therapy is appealing, we are not suggesting that the HER-2+ or luminalHer2+ subgroups be preferentially treated by mastectomy. Randomized trials have demonstrated that the addition of trastuzumab to chemotherapy decreases local failure by approximately $50 \%$ compared with treatment with chemotherapy alone, so it is likely that rates of local failure in these groups have decreased [9]. In addition, Kyndi et al. [4] examined the risks of locoregional recurrence after mastectomy with and without radiotherapy based on the subtype classification, and they found higher rates of failure in HER-2+ than others. Collectively, these findings suggest that HER-2 status (in the absence of trastuzumab treatment) is a poor prognostic factor but is still not predictive of appropriate local therapy.

Our findings support those of Wiechmannet al. [21], showing that after controlling for patient age, tumor size, and grade, TNBC had an OR of 0.7 for one or more positive nodes and 0.8 for $\geq 4$ metastatic lymph nodes. The relative likelihood of lymph node involvement, which is considered a poor prognostic indicator, is lower for TNBC compared to other breast cancer subtypes in four or more metastatic lymph nodes. It is suggested by this finding that the aggressive nature of TNBC is not directly associated with lymphatic spread, and it is consistent with the findings of other studies [7, 18, 21-24].

Tumor size was known as the most significant predictor of LN metastases [25, 26]. Similarly, our study demonstrated tumor size as a significant independent predictive factor for positive LN status with an odds ratio of 2.3 for T2 vs. T1 tumors and 2.7 for T3 vs. T1 tumors. In other studies, Mustafa et al. [27], when reviewing more than 2100 patients with $\leq 1 \mathrm{~cm}$ invasive tumors, show by multivariate analyses that not only the size of the tumor, but also the grade of the tumor and the patient's age are related to the incidence of nodal involvement. However, we only found that in tumors of all sizes, higher grade was predictive of nodal positivity. Age is not associated with increased likelihood of nodal metastases [25, 26, 28]. 


\section{Conclusions}

The results of our study are unexpected which suggest predictors of LN metastases only include higher grade and larger tumor size, and breast cancer subtype is not a statistically significant predictor of LN positivity. Even so, this information may still be useful in clinical practice. We found tumors overexpressing HER-2 (luminalHer2+ and HER-2+) and TNBC subtypes were more likely in grade 3 and T3 and HER-2+ tumors were associated more frequently with involved nodes. These are beneficial to make decisions regarding neoadjuvant therapy, breast-conserving therapy, axillary surgery, and locoregional radiation.

\section{Competing interests}

The authors declare that they have no competing interests.

\section{Authors' contributions}

LYH drafted the manuscript and designed as well as collected data and clinical records of the patients. CED and CYF helped to edit and revise the manuscript. PCM and YF helped to collect the data and clinical records of the patients. WOC and $\mathrm{ZXH}$ made up the surgical team handling most of the patients. All authors read and approved the final manuscript.

\section{Authors' information}

LYH is a graduate of Wenzhou Medical University, Wenzhou, Zhejiang, People's Republic of China. WOC is Chief Physician of the Department of Oncology, The First Affiliated Hospital of Wenzhou Medical University, Wenzhou, Zhejiang, People's Republic of China. CED is a surgeon of the Department of Oncology, The First Affiliated Hospital of Wenzhou Medical University, Wenzhou, Zhejiang, People's Republic of China. CYF is a graduate of Wenzhou Medical University, Wenzhou, Zhejiang, People's Republic of China. PCM is a graduate of Wenzhou Medical University, Wenzhou, Zhejiang, People's Republic of China. YF is a graduate of Wenzhou Medical University, Wenzhou, Zhejiang, People's Republic of China. ZXH is Chief Physician of the Department of Oncology, The First Affiliated Hospital of Wenzhou Medical University, Wenzhou, Zhejiang, People's Republic of China.

\section{Acknowledgements}

We thank all of the pathologists at the First Affiliated Hospital of Wenzhou Medical University for their assistance with the pathologic analysis. Without their efforts, this article would not be possible. This work was supported by the National Natural Science Foundation of China (NO.81372380) and Medical Scientific Research Foundation of Zhejiang Province (NO.2011KYA108).

Received: 12 June 2015 Accepted: 28 July 2015

Published online: 15 August 2015

\section{References}

1. Perou CM, Sorlie T, Eisen MB, van de Rijn M, Jeffrey SS, Rees CA, et al. Molecular portraits of human breast tumours. Nature. 2000;406(6797):747-52

2. Sorlie T, Perou CM, Tibshirani R, Aas T, Geisler S, Johnsen H, et al. Gene expression patterns of breast carcinomas distinguish tumor subclasses with clinical implications.

Proc Natl Acad Sci USA. 2001;98:10869-74

3. Nguyen PL, Taghian AG, Katz MS, Niemierko A, Abi Raad RF, Boon WL, et al. Breast cancer subtype approximated by estrogen receptor, progesterone receptor, and HER-2 is associated with local and distant recurrence after breast-conserving therapy. J Clin Oncol. 2008;26:2373-8.

4. Kyndi M, Overgaard M, Nielsen HM, Sørensen FB, Knudsen H, Overgaard J. High local recurrence risk is not associated with large survival reduction after postmastectomy radiotherapy in high-risk breast cancer: a subgroup analysis of DBCG 82 b\&c. Radiother Oncol. 2009:90:74-9.

5. Bevilacqua JLB, Kattan MW, Fey JV, Cody 3rd HS, Borgen PI, Van Zee KJ. Doctor, what are my chances of having a positive sentinel node? A validated nomogram for risk estimation. J Clin Oncol. 2007;25(24):3670-9.
6. Yoshihara E, Smeets A, Laenen A, Reynders A, Soens J, Van Ongeval C. Predictors of axillary lymph node metastases in early breast cancer and their applicability in clinical practice. Breast. 2013;22(3):357-61.

7. Hwang RF, Krishnamurthy S, Hunt KK, Mirza N, Ames FC, Feig B, et al. Clinicopathologic factors predicting involvement of nonsentinel axillary nodes in women with breast cancer. Ann Surg Oncol. 2003;10(3):248-54

8. Van Zee KJ, Kattan MW. Validating a predictive model for presence of additional disease in the non-sentinel lymph nodes of a woman with sentinel node positive breast cancer. Ann Surg Oncol. 2007;14(8):2177-8.

9. Sotiriou C, Neo S-Y, McShane LM, Korn EL, Long PM, Jazaeri A, et al. Breast cancer classification and prognosis based on gene expression profiles from a population-based study. Proc Natl Acad Sci USA. 2003;100(18):10393-8.

10. Coates A S. PG 0.1 Evolution of the St.Gallen Consensus process for the optimal treatment of women with breast cancer[J]. Breast, 2015, 24:S1.

11. Goldhirsch A, Winer EP, Coates AS, Gelber RD, Piccart-Gebhart M, Thurlimann B, et al. Personalizing the treatment of women with early breast cancer: highlights of the St Gallen International Expert Consensus on the Primary Therapy of Early Breast Cancer 2013. Ann Oncol. 2013;24:2206-23.

12. Sorlie T, Tibshirani R, Parker J, Hastie T, Marron JS, Nobel A, et al. Repeated observation of breast tumor subtypes in independent gene expression data sets. Proc Natl Acad Sci USA. 2003;100:8418-23.

13. Piccart-Gebhart MJ, Procter M, Leyland-Jones B, Goldhirsch A, Untch M, Smith I, et al. Trastuzumab after adjuvant chemotherapy in HER2-positive breast cancer. N Engl J Med. 2005;353:1659-72.

14. Romond EH, Perez EA, Bryant J, Suman VJ, Geyer CE Jr, Davidson NE, et al. Trastuzumab plus adjuvant chemotherapy for operable HER2-positive breast cancer. N Engl J Med. 2005;353:1673-84.

15. Gangi A, Chung A, Mirocha J, Liou DZ, Leong T, Giuliano AE. Breast-conserving therapy for triple-negative breast cancer. JAMA Surg. 2014;149(3):252-8.

16. Ragage F, Debled M, MacGrogan G, Brouste V, Desrousseaux M, Soubeyran I, et al. Is it useful to detect lymphovascular invasion in lymph node-positive patients with primary operable breast cancer? Cancer. 2010;116(13):3093-101.

17. Rivadeneira DE, Simmons RM, Christos PJ, Hanna K, Daly JM, Osborne MP. Predictive factors associated with axillary lymph node metastases in T1a and T1b breast carcinomas: analysis in more than 900 patients. J Am Coll Surg. 2000;191(1):1-6. discussion 6-8.

18. Crabb SJ, Cheang MC, Leung S, Immonen T, Nielsen TO, Huntsman DD, et al. Basal breast cancer molecular subtype predicts for lower incidence of axillary lymph node metastases in primary breast cancer. Clin Breast Cancer. 2008:8:249-56.

19. Morrow M, Schmidt R, Hassett C. Patient selection for breast conservation therapy with magnification mammography. Surgery. 1995;118:621-6.

20. Buzdar AU, Valero V, Ibrahim NK, Francis D, Broglio KR, Theriault RL, et al. Neoadjuvant therapy with paclitaxel followed by 5-fluorouracil, epirubicin, and cyclophosphamide chemotherapy and concurrent trastuzumab in human epidermal growth factor receptor 2-positive operable breast cancer: an update of the initial randomized study population and data of additional patients treated with the same regimen. Clin Cancer Res. 2007;13:228-33.

21. Wiechmann L, Sampson M, Stempel M, Jacks LM, Patil SM, King T, et al. Presenting features of breast cancer differ by molecular subtype. Ann Surg Oncol. 2009;16(10):2705-10

22. Reyal F, Rouzier R, Depont-Hazelzet B, Bollet MA, Pierga JY, Alran S, et al. The molecular subtype classification is a determinant of sentinel node positivity in early breast carcinoma. PLoS ONE. 2011;6(5):e20297.

23. Freedman GM, Anderson PR, Li T, Nicolaou N. Locoregional recurrence of triple-negative breast cancer after breast-conserving surgery and radiation. Cancer. 2009;115(5):946-51.

24. Dent R, Trudeau M, Pritchard Kl, Hanna WM, Kahn HK, Sawka CA, et al. Triple-negative breast cancer: clinical features and patterns of recurrence. Clin Cancer Res. 2007;13(15 Pt 1):4429-34.

25. Viale G, Zurrida S, Maiorano E, Mazzarol G, Pruneri G, Paganelli G, et al. Predicting the status of axillary sentinel lymph nodes in 4351 patients with invasive breast carcinoma treated in a single institution. Cancer. 2005;103(3):492-500.

26. Silverstein MJ, Skinner KA, Lomis TJ. Predicting axillary nodal positivity in 2282 patients with breast carcinoma. World J Surg. 2001;25(6):767-72.

27. Mustafa IA, Cole B, Wanebo HJ, Bland KI, Chang HR. The impact of histopathology on nodal metastases in minimal breast cancer. Arch Surg. 1997;132(4):384-90. discussion 390-1.

28. Harden SP, Neal AJ, Al-Nasiri N, Ashley S, Querci della Rovere G. Predicting axillary lymph node metastases in patients with $\mathrm{T} 1$ infiltrating ductal carcinoma of the breast. Breast. 2001;10(2):155-9. 